Supporting Information

\title{
Anomalous, Multi-Stage Liquid Water Diffusion and Ionomer Swelling Kinetics in Nafion and Nafion Nanocomposites
}

\author{
Apoorv Balwani and Eric M. Davis* \\ Department of Chemical and Biomolecular Engineering, Clemson University, Clemson, SC \\ 29634
}

*To whom correspondence should be addressed; Email: ericd@clemson.edu 


\section{Multistage Sorption Kinetics from Time-Resolved Attenuated Total Reflectance-Fourier Transform Infrared (tATR-FTIR) Spectroscopy}

Liquid water sorption through both Nafion and Nafion nanocomposites containing silica nanoparticles (SiNPs) was captured using $t$ ATR-FTIR spectroscopy, where spectra at the polymerATR crystal interface were collected at regular time intervals after the membranes were challenged with liquid water at the opposite face (i.e., the polymer-water interface). This process was automated using the OMNIC ${ }^{\text {тм }}$ Series Software (Thermo Fisher Scientific), which was paired with a Fourier transform infrared (FT-IR) spectrometer (Nicolet iS50R FT-IR spectrometer; Thermo Scientific). For these experiments, individual spectra were collected every $\approx 0.78 \mathrm{~s}$ ( 2 scans per

(a)

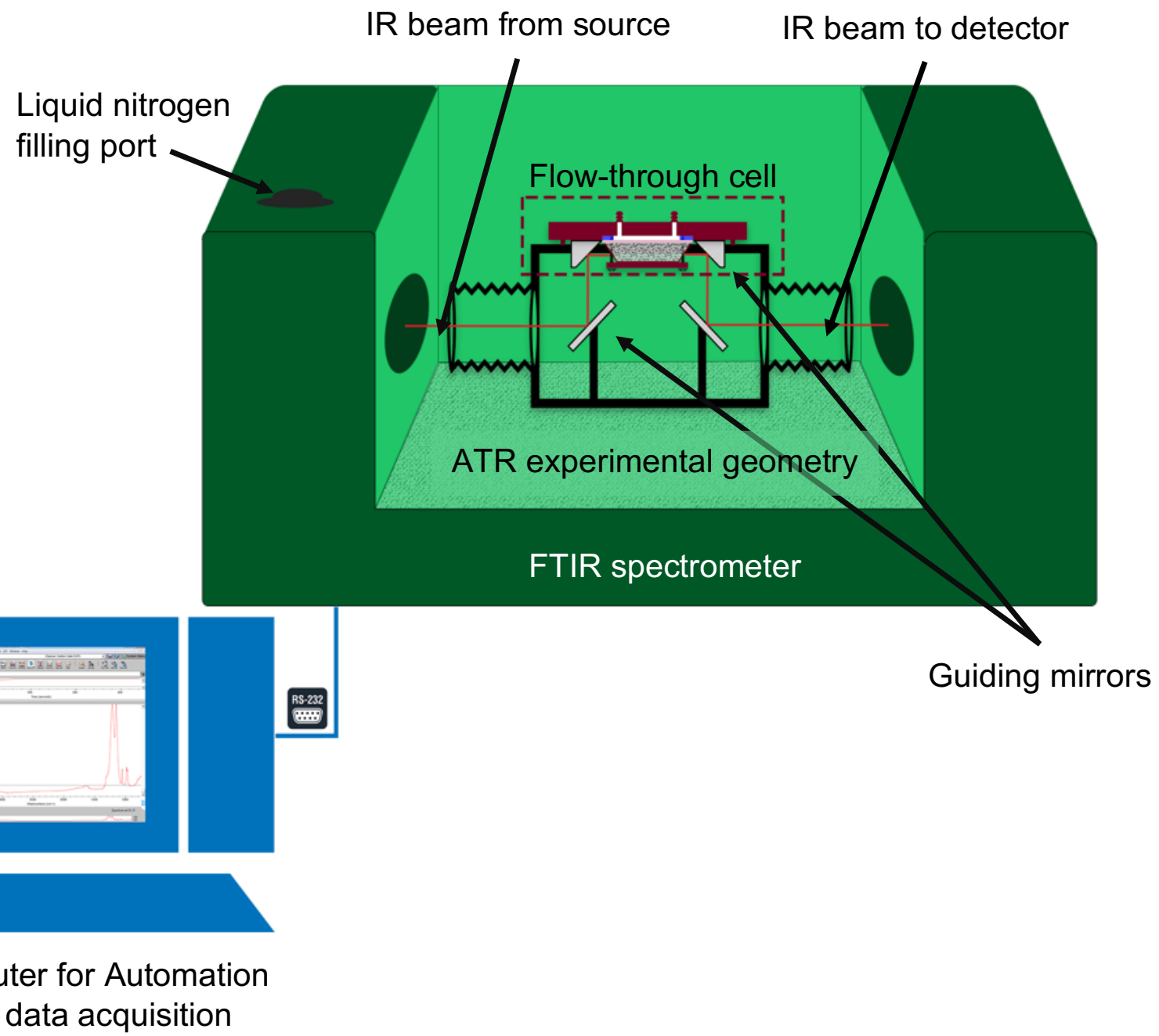


(b)

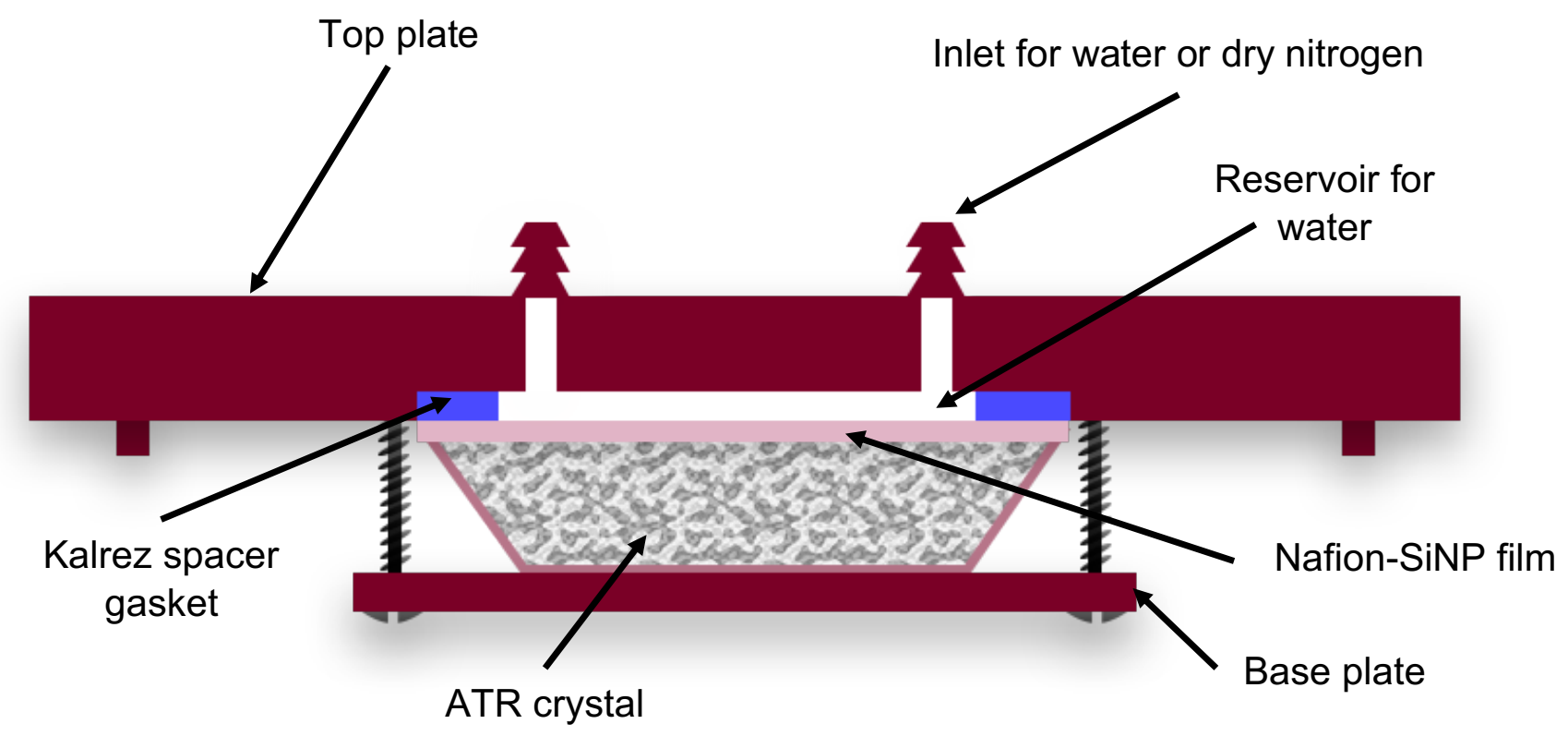

(c)

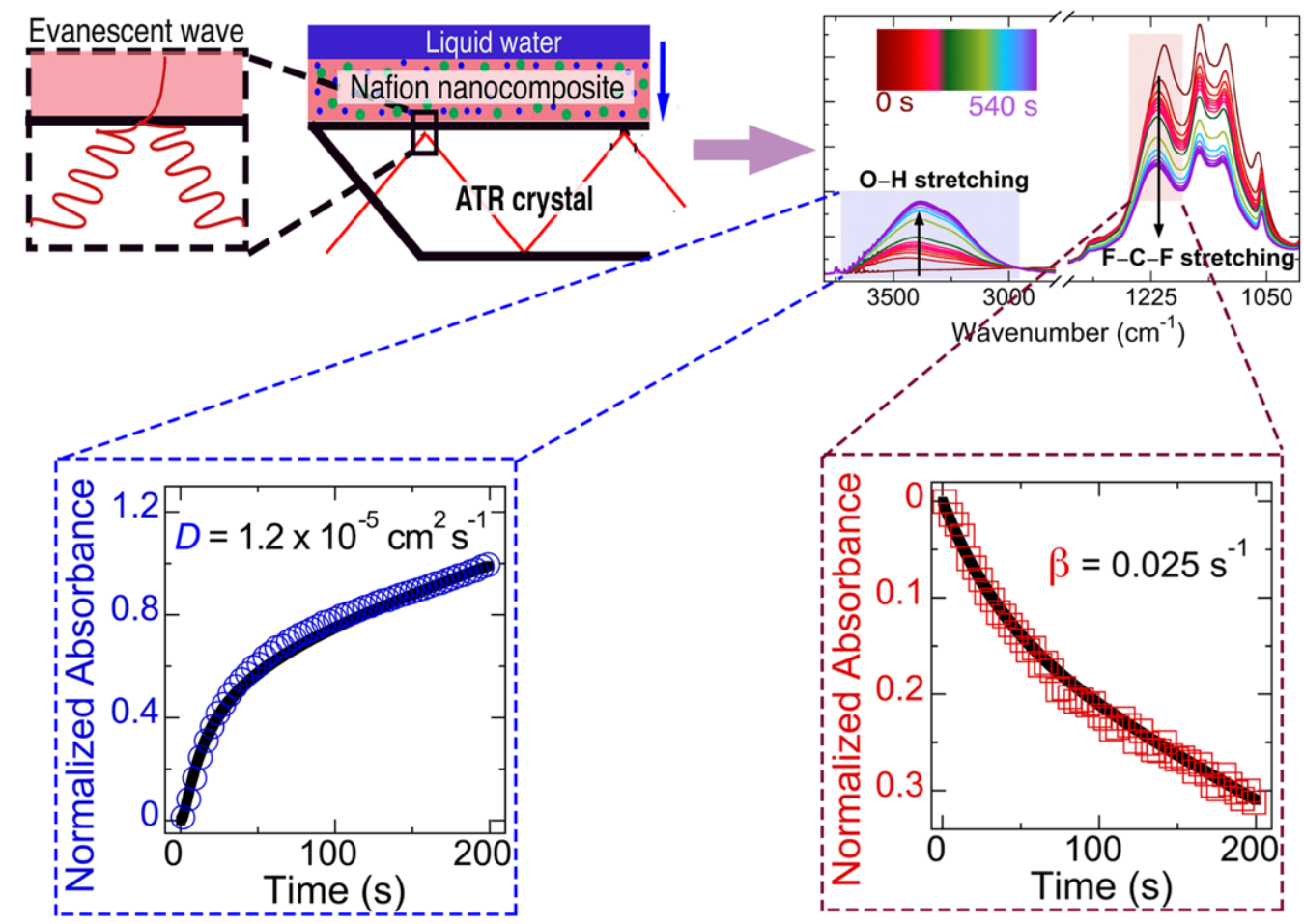

Figure S1. (a) Schematic layout of the time-resolved ATR-FTIR spectroscopy experiments to study hydration in dry Nafion-SiNP films, (b) magnified schematic of the flow-through cell assembly and (c) illustration of the liquid water hydration experiment in Nafion-SiNP films as captured by the ATR assembly. 
spectrum at a resolution of $4 \mathrm{~cm}^{-1}$ ), for experimental time periods ranging from a few hundred seconds to an hour. The schematic layout of the $t$ ATR-FTIR experimental setup is shown in Figure S1. Figure S1a presents the entire layout, while Figures S1b-c are included to detail the physical geometry of these transport experiments and the arrangement of different boundary layers. Please note that these schemes are very reductive, not to scale with the actual setup, and are for visualization purposes only.

Figures S2 - S6 show the liquid water uptake kinetics (represented by the normalized, integrated absorbance of the $\mathrm{O}-\mathrm{H}$ stretching peak with time) and the concomitant polymer swelling kinetics (represented by the scaled, integrated absorbance of the $\mathrm{F}-\mathrm{C}-\mathrm{F}$ stretching peak with time) for Nafion and for Nafion nanocomposites containing $4 \mathrm{wt} \%$ and $10 \mathrm{wt} \% \mathrm{SiNPs}$ at $25{ }^{\circ} \mathrm{C}$. Here, the $\mathrm{O}-\mathrm{H}$ absorbance data were normalized to the final uptake values, i.e., the absorbance value at the final time point. Note, a different version of Figure S4 (liquid water diffusion in annealed Nafion containing $4 \mathrm{wt} \%$ SiNPs) is presented in the main body of the manuscript (see Figure 4) for a more detailed analysis. For clarity, only 50\% of the data are shown in each Figure. Further, the shaded areas in the plots have been added to highlight the first stage of water sorption kinetics. The water uptake and polymer swelling kinetics of the first stage for each membrane were regressed to the diffusion-relaxation model (see eq 10), and the results of this analysis are presented in Table 1 of the manuscript. 


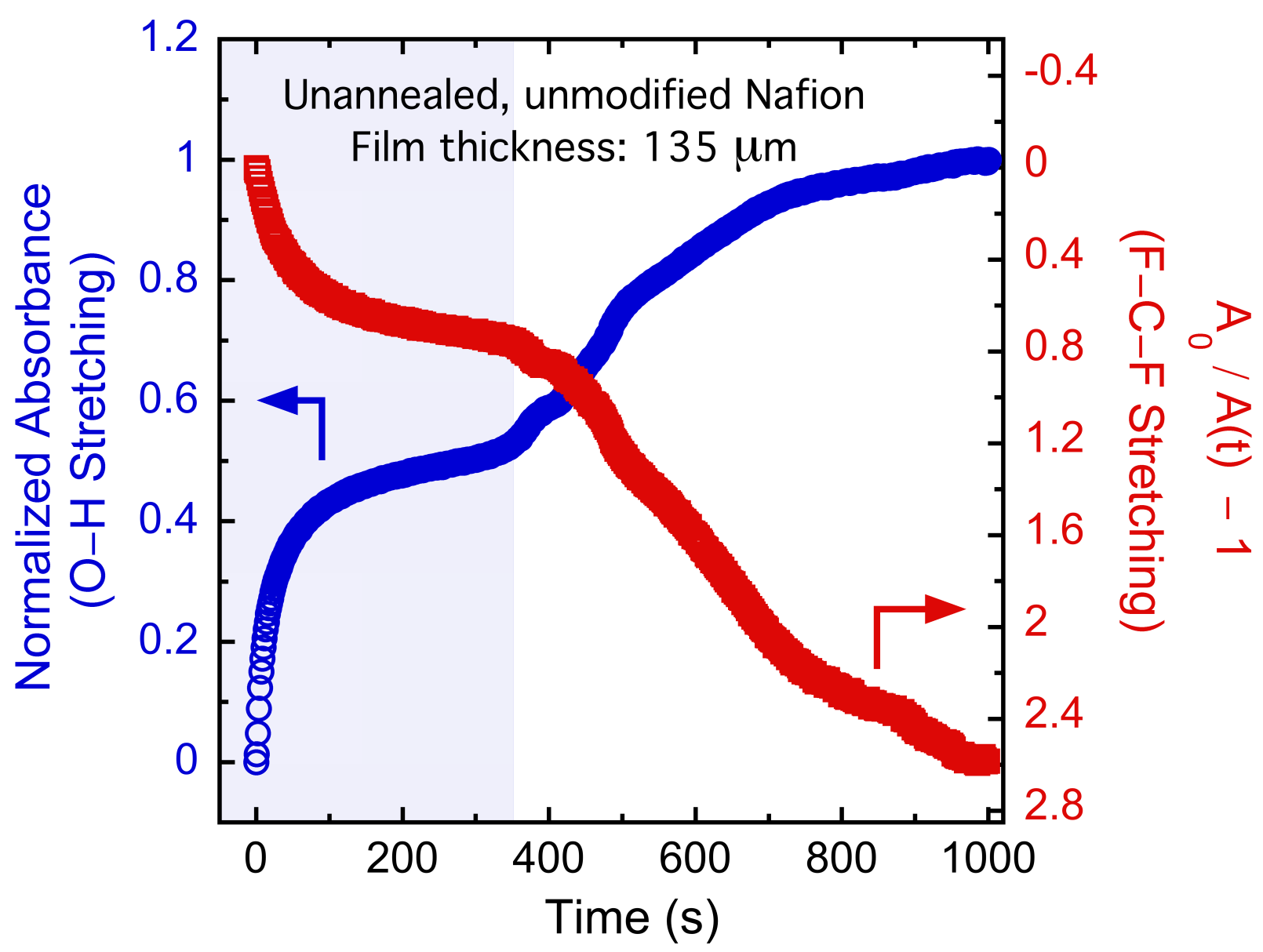

Figure S2. Time-resolved, normalized $\mathrm{O}-\mathrm{H}$ stretching (open blue circles) and $\mathrm{F}-\mathrm{C}-\mathrm{F}$ stretching (open red squares) absorbance data of liquid water diffusion in unannealed, unmodified Nafion (i.e., Nafion containing no SiNPs) at $25^{\circ} \mathrm{C}$. 


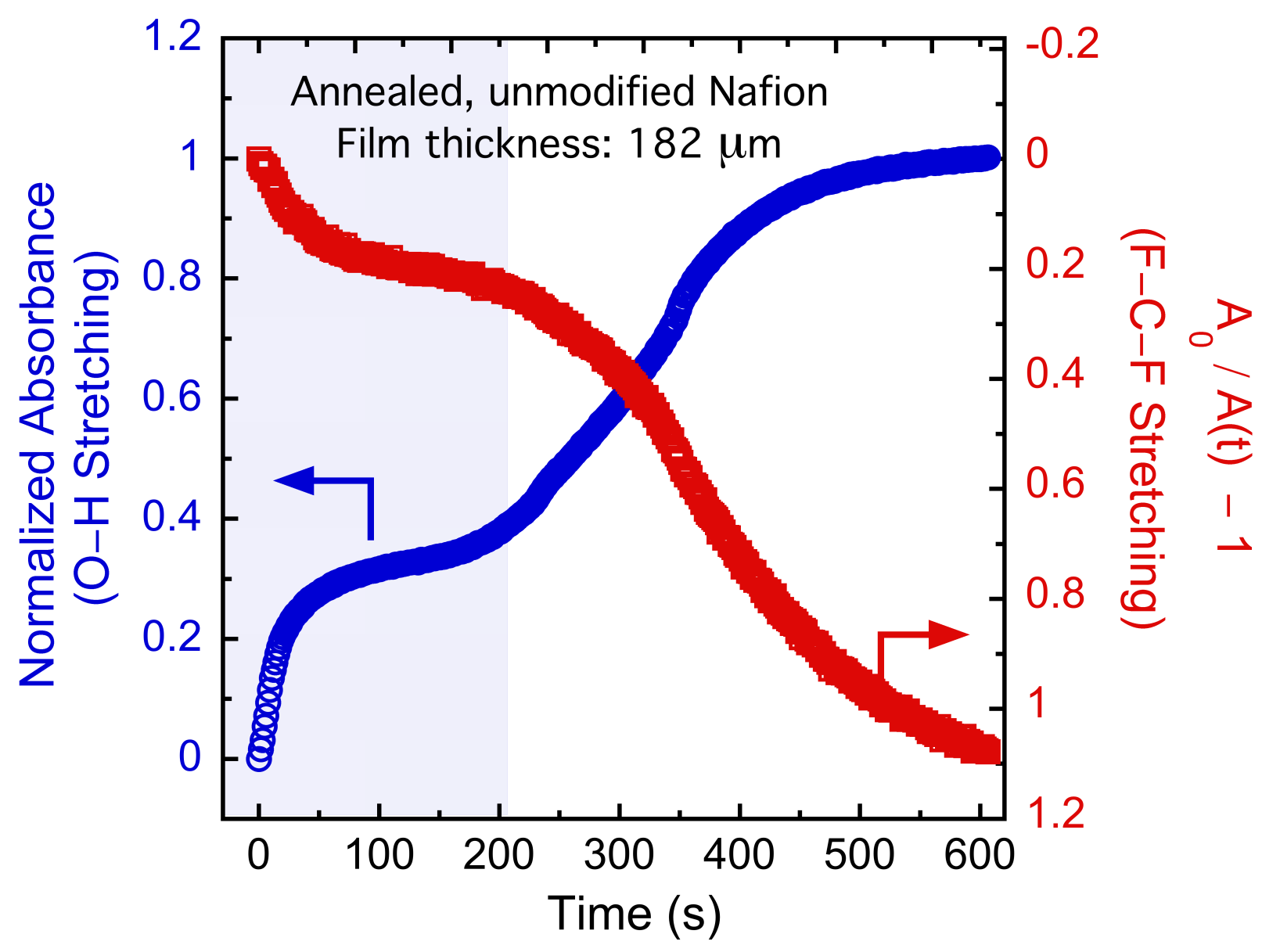

Figure S3. Time-resolved, normalized $\mathrm{O}-\mathrm{H}$ stretching (open blue circles) and $\mathrm{F}-\mathrm{C}-\mathrm{F}$ stretching (open red squares) absorbance data of liquid water diffusion in annealed, unmodified Nafion (i.e., Nafion containing no SiNPs) at $25^{\circ} \mathrm{C}$. 


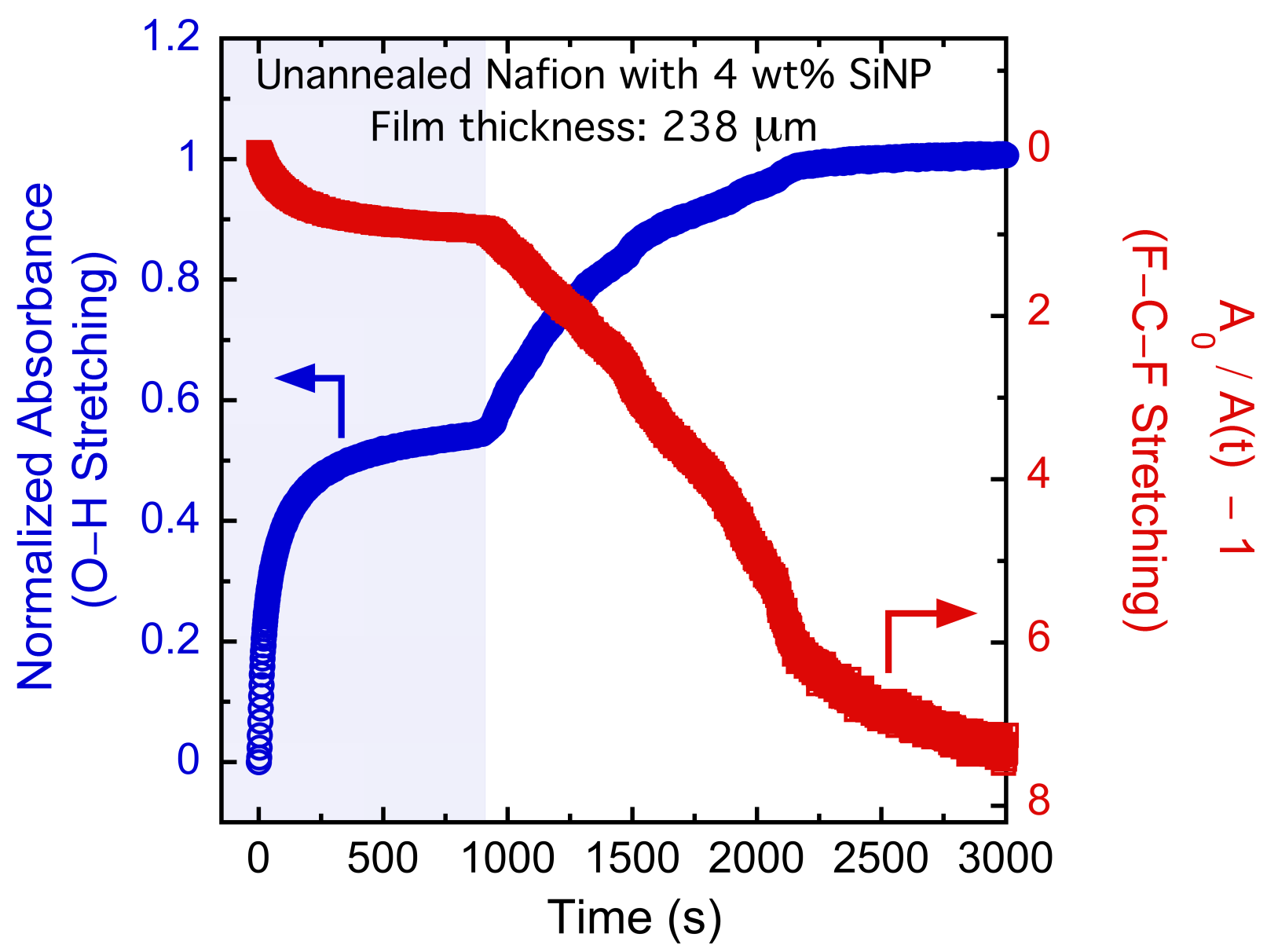

Figure S4. Time-resolved, normalized $\mathrm{O}-\mathrm{H}$ stretching (open blue circles) and $\mathrm{F}-\mathrm{C}-\mathrm{F}$ stretching (open red squares) absorbance data of liquid water diffusion in unannealed, Nafion containing $4 \mathrm{wt} \%$ SiNPs at $25^{\circ} \mathrm{C}$. 


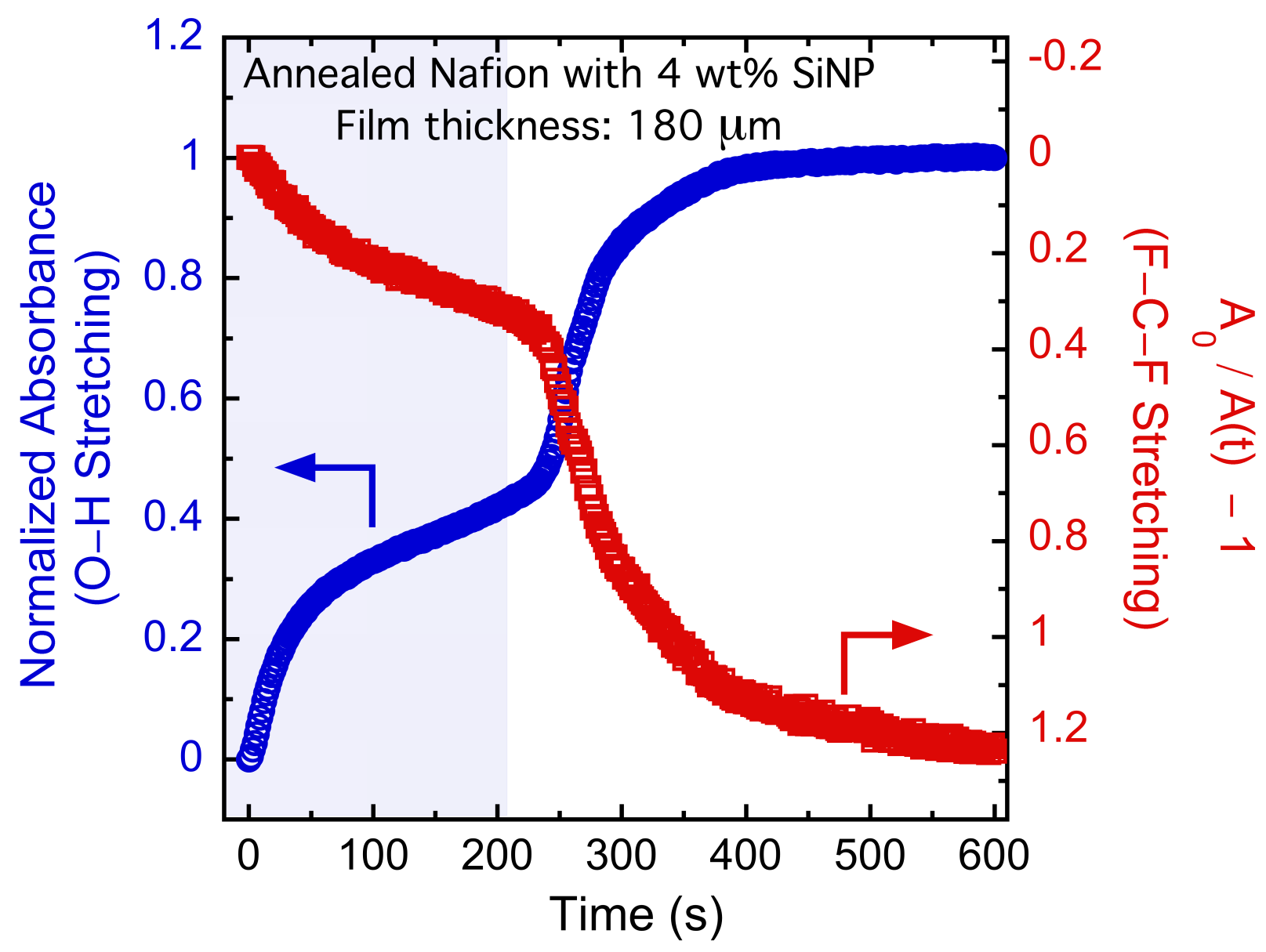

Figure S5. Time-resolved, normalized $\mathrm{O}-\mathrm{H}$ stretching (open blue circles) and $\mathrm{F}-\mathrm{C}-\mathrm{F}$ stretching (open red squares) absorbance data of liquid water diffusion in annealed, Nafion containing $4 \mathrm{wt} \%$ SiNPs at $25^{\circ} \mathrm{C}$. 


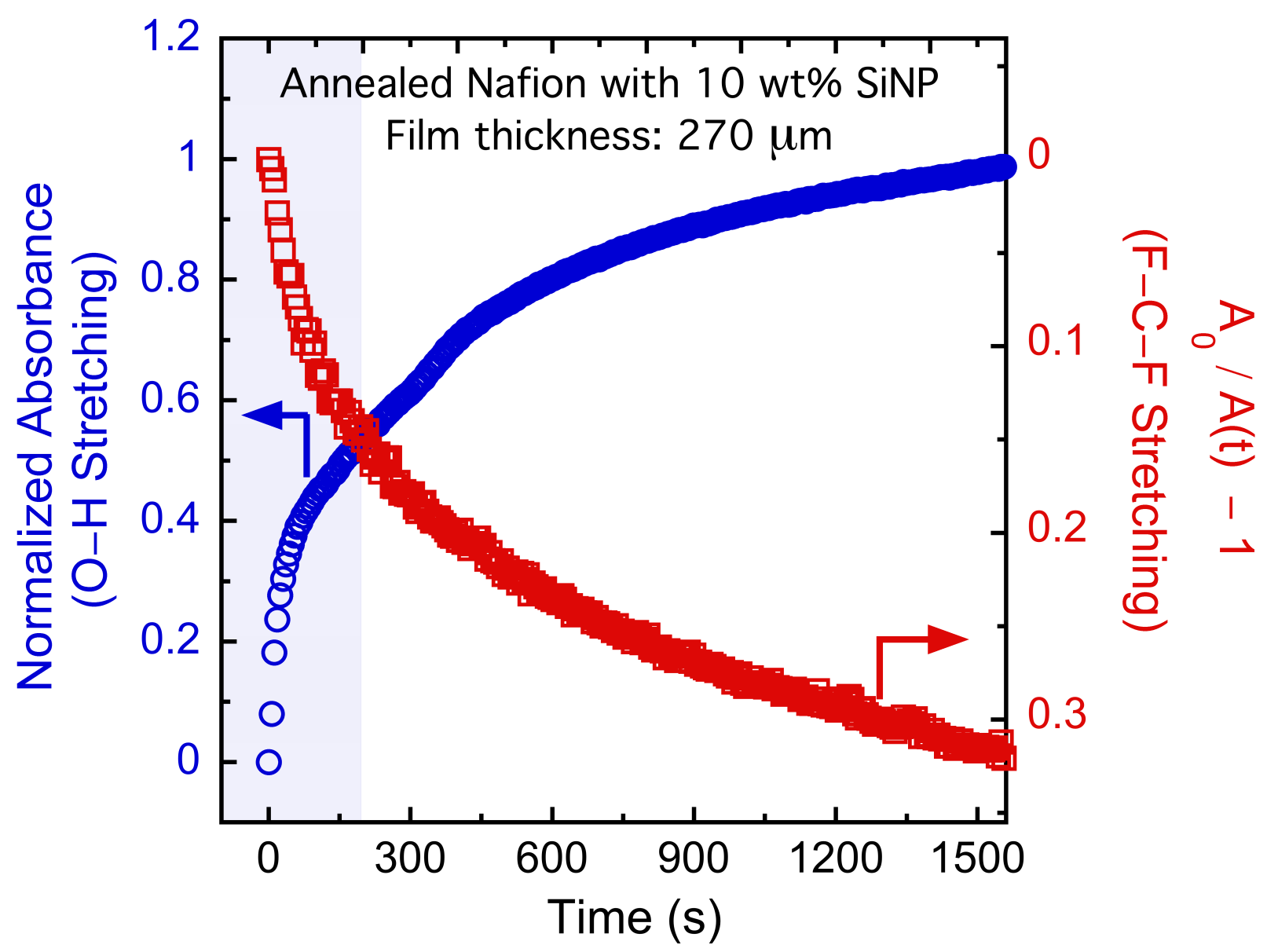

Figure S6. Time-resolved, normalized $\mathrm{O}-\mathrm{H}$ stretching (open blue circles) and $\mathrm{F}-\mathrm{C}-\mathrm{F}$ stretching (open red squares) absorbance data of liquid water diffusion in annealed, Nafion containing $10 \mathrm{wt} \%$ SiNPs at $25^{\circ} \mathrm{C}$. 